\title{
Tree Extraction of Airborne LiDAR Data Based on Coordinates of Deep Learning Object Detection from Orthophoto over Complex Mangrove Forest
}

\author{
Alvin Sarraga Alon ${ }^{1}$, Enrique D. Festijo ${ }^{2}$, Cherry D. Casuat ${ }^{3}$ \\ ${ }^{1}$ Technological Institute of the Philippines, Philippines, aalon.cpe@tip.edu.ph \\ ${ }^{2}$ Technological Institute of the Philippines, Philippines, enrique.festijo@tip.edu.ph \\ ${ }^{3}$ Technological Institute of the Philippines, Philippines, ccasuat.cpe@tip.edu.ph
}

\begin{abstract}
Knowing rainforest environments is rendered challenging by distance, vegetation intensity, and coverage; however, knowing the complexity and sustainability of these landscapes is important for ecologists and conservationists. The airborne light detection and ranging (LiDAR) system has made dramatic improvements to forest data collection and management especially on the forest inventory aspect. LiDAR can reliably calculate tree-level characteristics such as crown scale and tree height as well as derived measures such as breast height diameter (DBH). To do this, an exact tree extraction method is needed inside LiDAR data. Within LiDAR data, tree extraction often starts by locating the treetops via local maxima (LM). Wide-ranging efforts have been developed to extract individual trees from LiDAR data by starting to localize treetops through LM within LiDAR data. Throughout this research, a demonstration of a new tree extraction framework inside LiDAR Point Cloud by incorporating a new tree extraction method using the bounding-box coordinates provided by deep learning-based object detection. Tree extraction inside the LiDAR point cloud using the bounding-box coordinates was successful and feasible.
\end{abstract}

Key words: Georeferencing, Las Clipping, LiDAR, Mangrove, Orthophoto, Tree Extraction.

\section{INTRODUCTION}

Classification of the forest environment at an individual tree level is becoming a critical need for many applications in forest management and ecology [1]. Individual tree information is often useful to update forest inventories and predict growth and yield, or also to classify trees with strong biodiversity values [2].

Forest field inventory is a well-established method that relies on awareness of forest layout and distribution to support forest analysis, monitoring, and management [3]. Field inventories are performed in field plots, where tree information (e.g. crown size, height, and trunk diameter) is typically obtained through individual tree level measures (e.g. plot-level inventory) [4]. Forest inventory fieldwork, however, is laborious and costly because field calculations entail a great deal of time and energy, thereby reducing the number of field inventories that can be obtained [5]. Ever after field inventory began, efforts to increase the field inventory performance have begun. Countless methods, equipment, and procedures have been developed but development has been slow until essentially a laser-based measurement device named Light Detection and Ranging (LiDAR) has become available [6].

Airborne light detection and range technology (LiDAR) has made significant advancements in forest data acquisition and management during the past two decades through the delivery of product data at exceptional spatial and temporal resolutions [7]. However, reliable tree-level attributes such as tree height and crown size, as well as derivative estimates such as breast height diameter (DBH for trunk diameter) are needed; volume; and LiDAR results involve biomass, precise tree extraction approaches [8]. Tree extraction is an essential process for the correct measurement of individual attributes at tree level.

Extensive efforts have been established to extract individual trees from LiDAR data by beginning to identify treetops from either the canopy height model (CHM) or LiDAR point clouds through local maxima (LM) [9]. Local maxima are used for crown segmentation, as reference points (or seed points). Since LM detection is vulnerable to commissioning (or incorrect detection), various methods tried to improve treetop recognition [10]. Which involves image filtering of fixed or variable dimension, multi-scale filtering, matching of models, stochastic geometry centered on specified point processes and the spatial contour form [11].

The tree crown extraction was generally accomplished after identification of the local maxima by implementing the watershed methods, fitting functions, wavelet analysis, region growing methods, graph-theoretical approach to tree crown 
delineation, non-parametric approach, and a template matching to segmenting trees [12]. Thus, tree extraction in a LiDAR point cloud and calculation of all other properties of the tree structure is feasible.

Nevertheless, the above-mentioned approaches indicate that there is no uniformly superior approach and that there are difficulties in extracting trees with overlapping tree crowns with varying heights and distribution with the diameter as the overlapping trees do not fulfill the principle of geometric restriction [13]. For illustration, crowns of the adjacent tree of identical heights and distribution of density can be wrongly identified as a single treetop. Instead, the local maxima nontreetop may be incorrectly interpreted as treetops and may impact the tree structure attributes that are vulnerable to errors [14]. This should also be noted that errors arising during single tree extraction are duplicated in subsequent stages of the study of tree attributes, thus the removal or at least reduction of such inaccuracies is of considerable significance.

Keeping in mind the difficult issues listed above, while most research extracts individual trees by identifying treetops from local maxima either from the CHM or from fragmented point clouds within the larger LiDAR point cloud, the study specifically addressed the individual tree extraction within the LiDAR point cloud through incorporating a different approach to detecting individual trees. In a Very High Spatial Resolution image (Orthophoto), which identified a specific tree genus, in particular, a deep learning technique for tree identification was used, using the boundary box coordinates of an identified tree from an orthophoto to extract the tree from a LiDAR point cloud. This makes it easier to measure the attributes of the tree structure starting from the treetop to its trunk.

In this study, the researcher used from [15] 's study a predetected specific genus of a tree within Orthophoto. [15] used a RetinaNet CNN architecture to analyze the Orthophoto to handle the density and diversity of trees found in forest canopies. Geographic information systems (GIS) were then used within LiDAR to extract trees. The researcher is hopeful from the combination of an Orthophoto and LiDAR Point Cloud that the study 's performance could turn out to be one of the tree LiDAR extraction standards in the future.

\section{DATASETS AND MATERIALS}

\subsection{Software}

Using Inertial Explorer, the three UAV sensor data - GNSS, LiDAR, and IMU - were post-processed. The trajectory of GNSS was carried out to generate a Smoothed Best Estimate of Trajectory (SBET) using the IMU information. The SBET was then used to create a calculated and georeferenced point cloud, along with the LiDAR field data.

A point cloud analysis software, TerraScan has been used to describe the cluster of measured objects. $\mathrm{R}$ and Geographic
Information Systems (GIS) ArcGIS software has been used for the extraction (LiDAR).

\subsection{Hardware}

Figure 1 shows the hardware used by the study such as LiDAR sensor, camera, and Unmanned Aerial Vehicle (UAV) or Drone.

- LiDAR Sensor. Phoenix AL3-32, a lightweight integrated LIDAR device that comprises an IMU, a GNSS processor, a laser scanner, and a micro-computer.

- Camera. To create Orthophoto, a 16 Megapixel highresolution RGB camera with an ISO scale of 100 25600 was used to capture aerial photography.

- Unmanned Aerial Vehicle (UAV) or Drone. The study used a DJI Matrice 600, a multi-rotor UAV with a full weight of $15.1 \mathrm{~kg}$ for take-off.

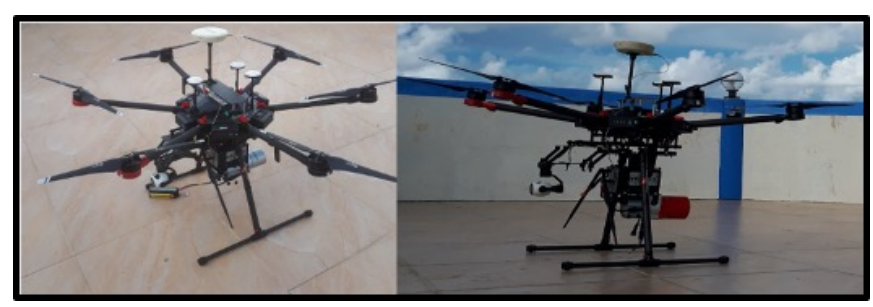

Figure 1: Hardware Setup

\subsection{Data}

The Field of Interest (FOI) is based in Palnab of Virac, Catanduanes, Philippines. The key data used in the study are the LiDAR and Orthophoto. During the same survey, it was taken that got the LiDAR data. The field of interest was segmented into sections. Figure 2 shows the FOI and mapping sites.

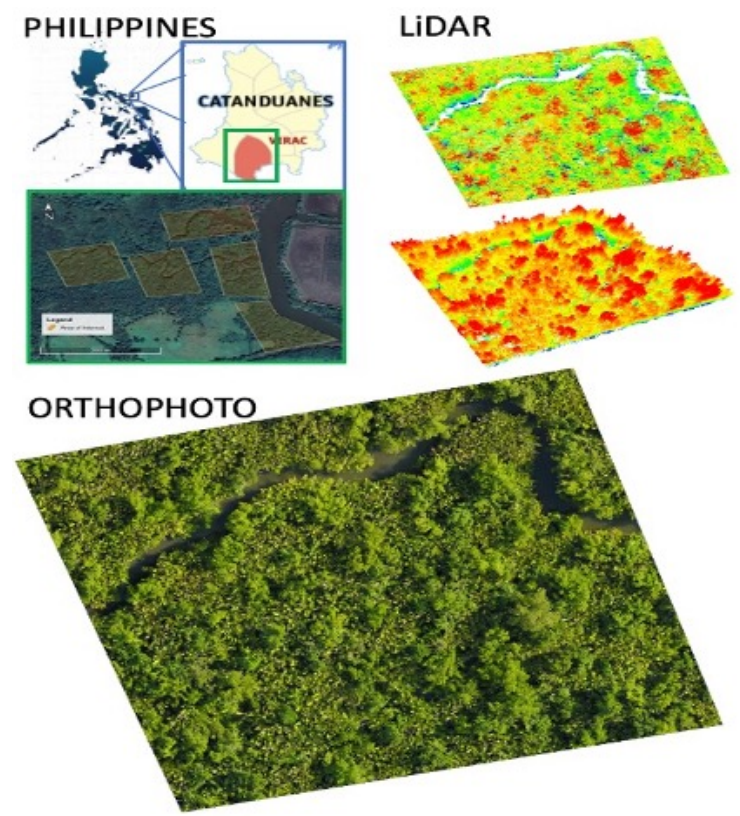

Figure 2: Field of Interest in Virac, Catanduanes, Philippines 


\section{METHODOLOGY}

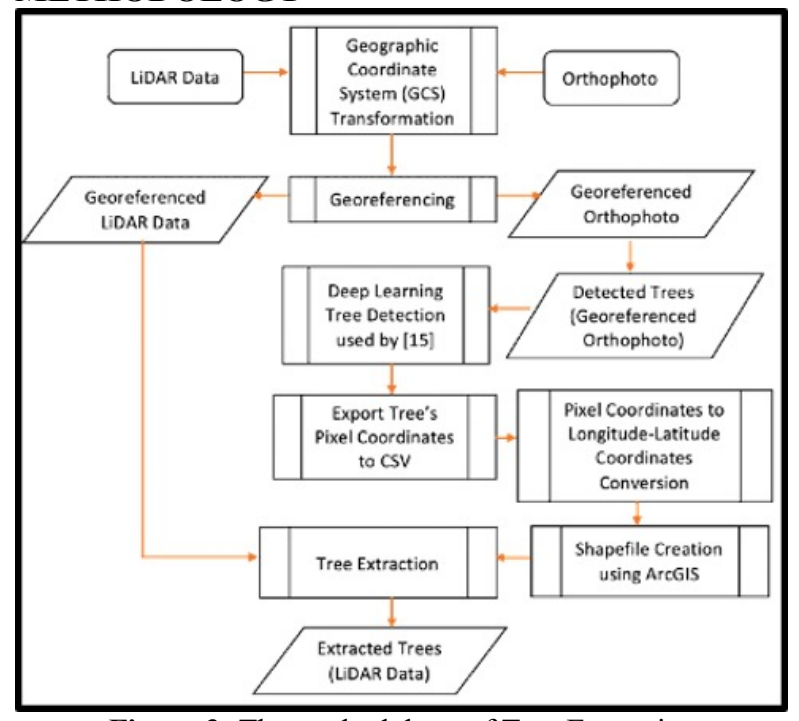

Figure 3: The methodology of Tree Extraction

\subsection{GCS Transformation}

Transformation of the Geographic Coordinates Systems (GCS) for both orthophoto and LiDAR was used to suit the coordinate systems of the data as seen in Table 1 . Without the matching of the LiDAR data and Orthophoto, difficulties and inaccuracies will occur in any analysis and mapping when performing on an incompatible data.

Table 1: GCS Transformation of Data

\begin{tabular}{lll}
\hline \hline Parameter & LiDAR Data & Orthophoto \\
\hline \multirow{2}{*}{ Coordinate System } & WGS_1984_UTM_Zone_51 & WGS_1984_UTM_Zone_5 \\
& $\mathrm{N}$ & $1 \mathrm{~N}$ \\
Central_Meridian & 123.00 & 123.00 \\
Scale_Factor & 0.99960 & 0.99960 \\
Latitude of Origin & 0.00 & 0.00 \\
False_Easting & 500000.00 & 500000.00 \\
False_Northing & 0.00 & 0.00 \\
\hline \hline
\end{tabular}

\subsection{Georeferencing}

When integrating data from various sources (e.g. LiDAR and Orthophoto) in GIS analysis, they must be properly matched with the support of geo-referencing. Because the raster output from the data consists of pixels, no position information is processed, so we cannot view, query, and analyze it with other geographic data unless it is geo-referenced as seen in Figure 4.

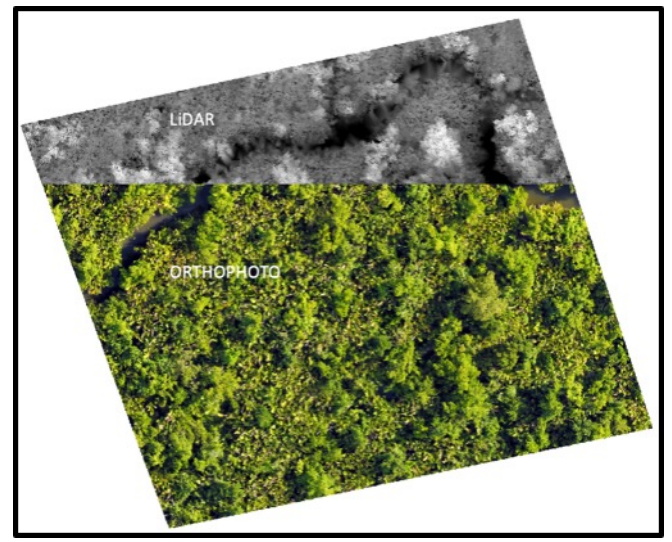

Figure 4: Georeferenced LiDAR and Orthophoto

\subsection{Pre-Detected Tree within Orthophoto}

In this analysis, the researcher used a pre-detected specific genus of a tree within Orthophoto from [15]'s study. Using RetinaNet CNN architecture, the Orthophoto was analyzed to tackle the height and variety of the trees used in forest canopies. The same study has been used by [16] - [18] that uses the power of machine vision deep learning.

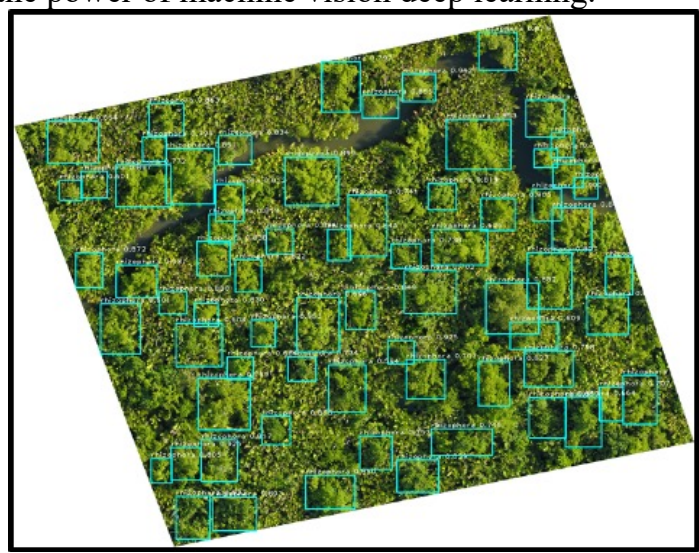

Figure 5: Pre-Detected Tree using the work of [15]

\subsection{Export Tree's Pixel Coordinates to CSV}

The CSV generated has a format of id,x1,y1,x2,y2 as shown in figure 6.

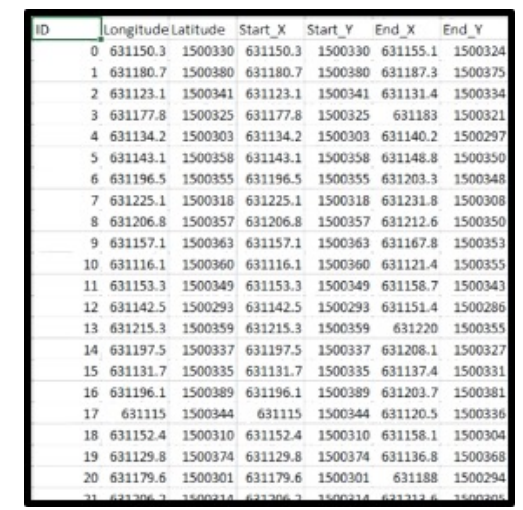

Figure 6: Exported CSV Pixel Coordinates

\subsection{Pixel to Longitude-Latitude Conversion}

After getting the pixel values, the study must get the equivalent longitude, latitude coordinates to crop these at the TIF images as shown in Figure 7.

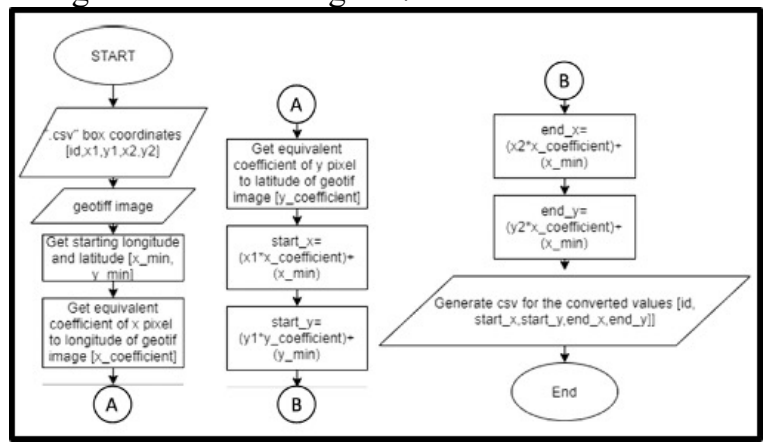

Figure 7: Conversion Algorithm 


\subsection{Shapefile Creation}

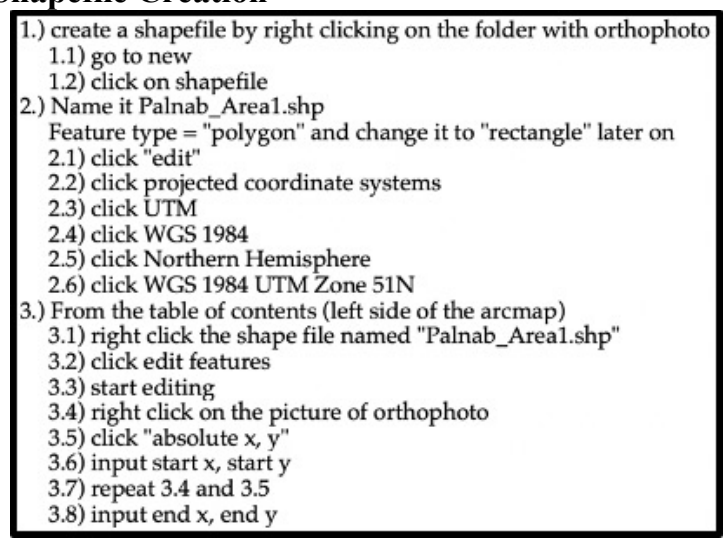

Figure 8: Creating the Shapefile Algorithm

After converting the pixel values, the study used the converted coordinates from the detected trees in a longitude-latitude format to create a shapefile. Figure 8 shows the shapefile creation algorithm using ArcGIS. Figure 9 below shows the shapefile output.

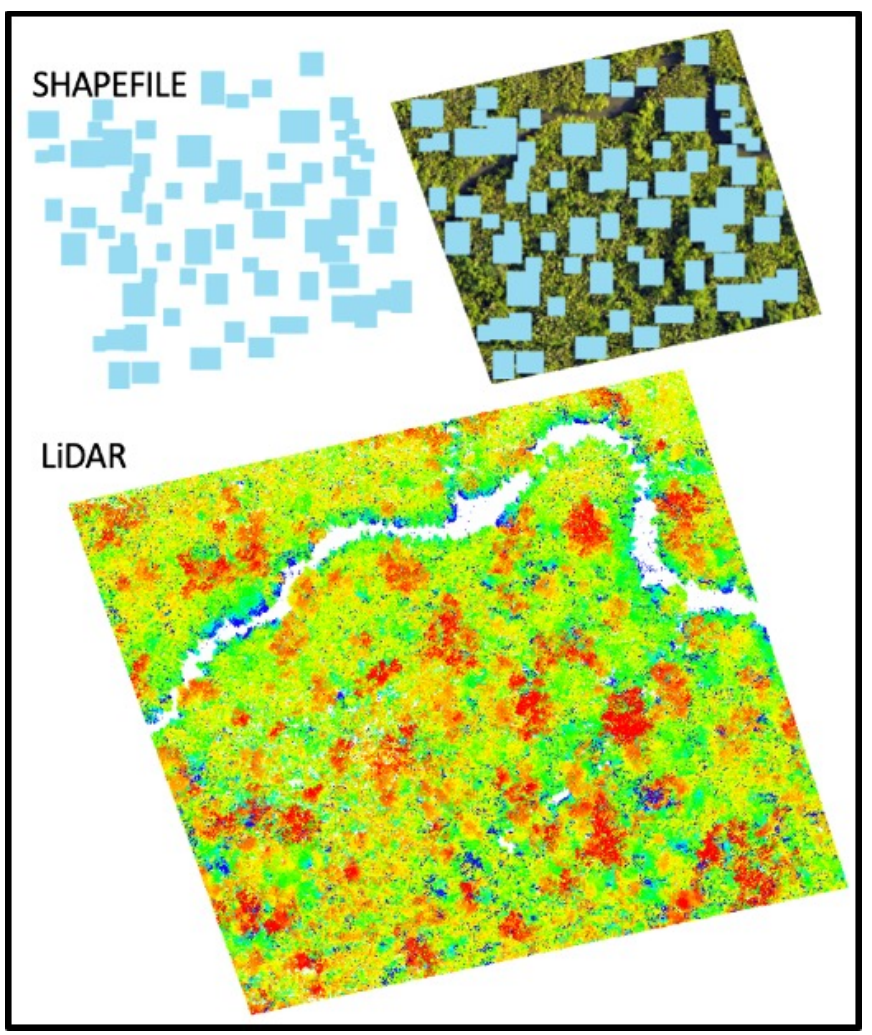

Figure 9: Shapefile Output on Orthophoto and LiDAR

\subsection{Results of Extracted Tree from LiDAR Data}

The shapefile was then used to extract all the LiDAR points associated with the detected trees using $\mathrm{R}$ as shown as an algorithm in Figure 10. Figure 9 also shows the LiDAR point cloud. Figure 11 shows the extracted LiDAR trees of each detected object from Figure 5.

1.) Use R programming to extract all the LiDAR as .las file. Using the following codes:
Install.packages("lidR")
2.) Location of the .las file to be extracted
ctg = catalog ("D:/MRSUAVE Dataset
072718/Mr.Suave_ClassifiedPointCloud/PALNAB/WGS84/Area3_Palnab_CLASSIFIEDLAS_-
WGS84.las")
3.) Location of the shape file created in ARCMAP
shp =
shapefile("D:/engrasa/CPE511/A1/Palnab_Orthophotos/Palnab_Orthophotos/PArea_3.shp")
4.) Location of .las file that has been extracted
opt_output_files(ctg) =
"D:/engrasa/CPE511/A1/Palnab_Orthophotos/Area3_Palnab_split_(ID)"
new_ctg<-lasclip(ctg, shp)
new_ctg

Figure 10: Tree Extraction Algorithm

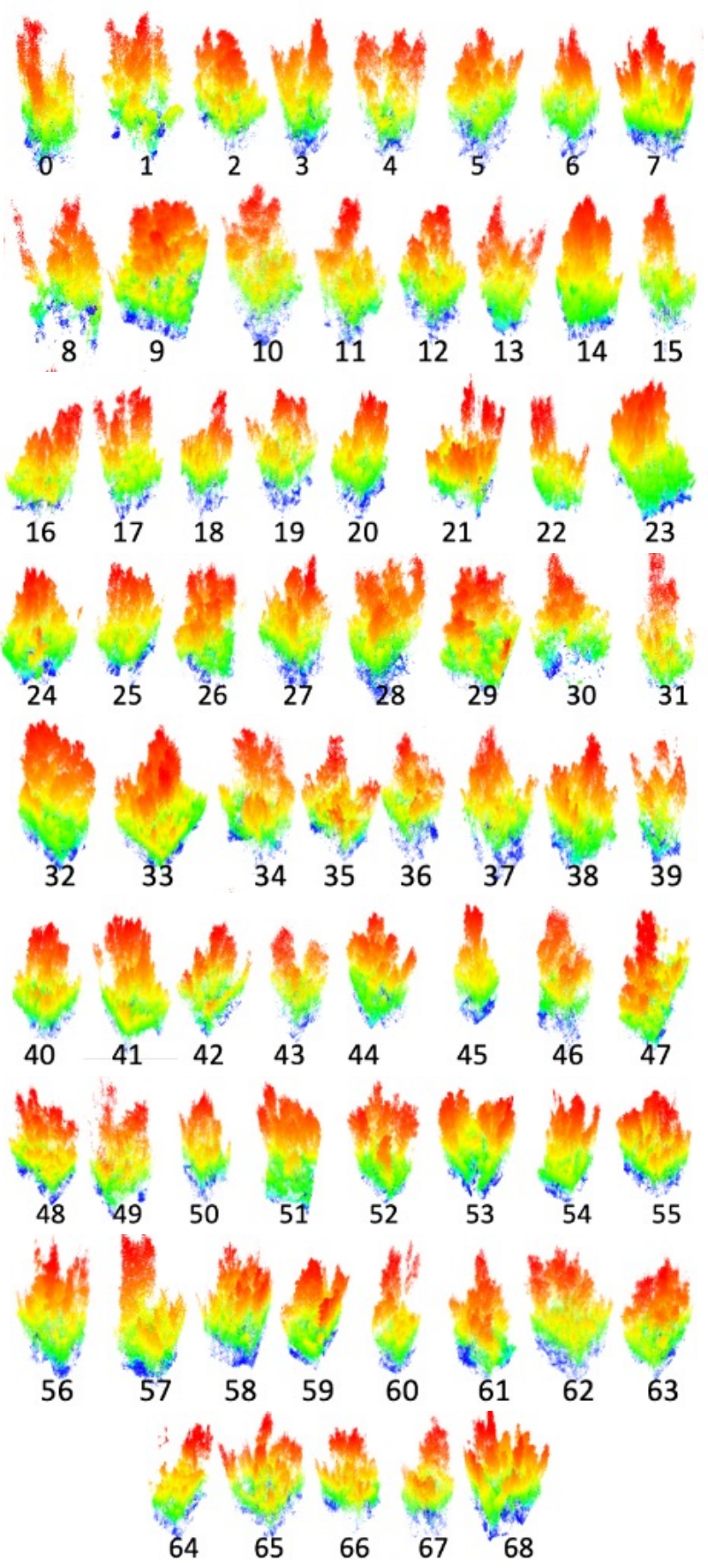

Figure 11: Extracted LiDAR Trees 


\section{CONCLUSION}

Rather than locating tree-tops utilizing local maxima (LM) for extraction inside LiDAR, the research established and proved a new approach to extracting a tree. The study implemented a new technique utilizing the coordinates of deep learning entity detection boundary boxes in a high-resolution image (orthophoto), then the analysis used the coordinates of the observed objects to extract them from the LiDAR point cloud. Trees were extracted by using the bounding box coordinates and proved its effectiveness as a new approach. Tree extraction within the LiDAR point cloud was successful, as shown in Figure 11. From the combination of an Orthophoto and LiDAR Point Cloud, the researcher is optimistic that the success of the study could turn out to be one of the LiDAR tree extraction benchmarks in the future.

\section{ACKNOWLEDGEMENT}

The researchers would like to convey their appreciation to the following: Commission on Higher Education (CHED) DARE TO Research Grant; MR-SUAVE Project (Mangrove RemoteSensing Unmanned Aerial Vehicle Explorer); and Catanduanes State University.

\section{REFERENCES}

1. S. Hummel and K. O'Hara, Forest Management, Encyclopedia of Ecology, pp. 1653-1662, 2008.

2. P. Bettinger, K. Boston, J. Siry and D. Grebner, Management of Forests and Other Natural Resources, Forest Management and Planning, pp. 1-20, 2017. doi: 10.1016/b978-0-12-809476-1.00001-1

3. C. Davis and R. Petersen, Tools for Monitoring Global Deforestation, Reference Module in Earth Systems and Environmental Sciences, 2016.

doi: 10.1016/b978-0-12-409548-9.09527-0

4. E. Tomppo, H. Olsson, G. Ståhl, M. Nilsson, O. Hagner and M. Katila, Combining national forest inventory field plots and remote sensing data for forest databases, Remote Sensing of Environment, vol. 112, no. 5, pp. 1982-1999, 2008. doi: 10.1016/j.rse.2007.03.032

5. S. Huang, C. Ramirez, M. McElhaney and K. Evans, F3: Simulating spatiotemporal forest change from field inventory, remote sensing, growth modeling, and management actions, Forest Ecology and Management, vol. 415-416, pp. 26-37, 2018.

doi: 10.1016/j.foreco.2018.02.026

6. M. Wulder et al., Lidar sampling for large-area forest characterization: A review, Remote Sensing of Environment, vol. 121, pp. 196-209, 2012. doi: 10.1016/j.rse.2012.02.001

7. M. Beland et al., On promoting the use of lidar systems in forest ecosystem research, Forest Ecology and Management, vol. 450, p. 117484, 2019.

doi: 10.1016/j.foreco.2019.117484

8. M. Palace et al., Estimating forest structure in a tropical forest using field measurements, a synthetic model and discrete return lidar data, Remote Sensing of Environment, vol. 161, pp. 1-11, 2015.

doi: 10.1016/j.rse.2015.01.020

9. J. Liu, J. Shen, R. Zhao and S. Xu, Extraction of individual tree crowns from airborne LiDAR data in human settlements, Mathematical and Computer Modelling, vol. 58, no. 3-4, pp. 524-535, 2013. doi: 10.1016/j.mcm.2011.10.071

10. M. Mohan et al., Optimizing individual tree detection accuracy and measuring forest uniformity in coconut (Cocos nucifera L.) plantations using airborne laser scanning, Ecological Modelling, vol. 409, p. 108736, 2019. doi: 10.1016/j.ecolmodel.2019.108736

11. F. Wagner et al., Individual tree crown delineation in a highly diverse tropical forest using very high resolution satellite images, ISPRS Journal of Photogrammetry and Remote Sensing, vol. 145, pp. 362377, 2018. doi: 10.1016/j.isprsjprs.2018.09.013

12. T. Dong, X. Zhang, Z. Ding and J. Fan, Multi-layered tree crown extraction from LiDAR data using graphbased segmentation, Computers and Electronics in Agriculture, vol. 170, p. 105213, 2020.

doi: 10.1016/j.compag.2020.105213

13. B. Weinstein, S. Marconi, S. Bohlman, A. Zare and E. White, Cross-site learning in deep learning RGB tree crown detection, Ecological Informatics, vol. 56, p. 101061, 2020. doi: 10.1016/j.ecoinf.2020.101061

14. J. Klingberg, J. Konarska, F. Lindberg, L. Johansson and S. Thorsson, Mapping leaf area of urban greenery using aerial LiDAR and ground-based measurements in Gothenburg, Sweden, Urban Forestry \& Urban Greening, vol. 26, pp. 31-40, 2017.

doi: $10.1016 /$ j.ufug.2017.05.011

15. A. S. Alon, E. D. Festijo and D. E. O. Juanico, Tree Detection using Genus-Specific RetinaNet from Orthophoto for Segmentation Access of Airborne LiDAR Data, 2019 6th IEEE International Conference on Engineering Technologies and Applied Sciences (ICETAS), Kuala Lumpur, Malaysia, 2019,(PrePrint)

16. A. Alon, A YOLOv3 Inference Approach for Student Attendance Face Recognition System, International Journal of Emerging Trends in Engineering Research, vol. 8 , no. 2, pp. 384-390, 2020. doi: $10.30534 /$ ijeter/2020/24822020

17. A. Alon, A Machine Vision Detection of Unauthorized On-Street Roadside Parking in Restricted Zone: An Experimental Simulated BarangayEnvironment, International Journal of Emerging Trends in Engineering Research, vol. 8, no. 4, pp. 1056-1061, 2020.

doi: $10.30534 /$ ijeter/2020/17842020

18. A. Alon, Machine Vision Recognition System for Iceberg Lettuce Health Condition on Raspberry Pi 4b: A Mobile Net SSD v2 Inference Approach, International Journal of Emerging Trends in Engineering Research, vol. 8, no. 4, pp. 1073-1078, 2020.doi: $10.30534 /$ ijeter/2020/20842020 